\title{
Different homogeneity detectors for improving Space-Time Adaptive Radar performance in heterogeneous clutter
}

\author{
Massimo Bertacca ${ }^{*}$ Douglas A. Gray ${ }^{* *}$, Luke Rosenberg ${ }^{* *}$ \\ *Dept. of Information Engineering, University of Pisa \\ Via Caruso 16, 56122 Pisa, ITALY \\ e-mail: massimo.bertacca@iet.unipi.it \\ ${ }^{* *}$ School of Electrical and Electronic Engineering- The University of Adelaide \\ North Terrace, 5005 Adelaide, SA-AUSTRALIA \\ e-mail: dgray@eleceng.adelaide.edu.au, luker@eleceng.adelaide.edu.au
}

\begin{abstract}
Secondary data selection for estimation of the clutter covariance matrix in space-time adaptive processing (STAP) is normally obtained from cells (range rings) in close proximity of the cell under test. The aim of this paper is the analysis of performance improvement of Space-Time Adaptive radars when secondary data selection is obtained by discriminating between quasi-homogeneous areas on the ground which generate clutter with different statistics (i.e. clutter edges including littoral, farmland-wooded hills or rural-urban interfaces). The algorithm presented in this paper, referred to as the Different Homogeneity Detector (DHD), has been tested with simulated data obtained by using a general clutter model and a uniform linear array.
\end{abstract}

\section{Introduction}

It is known that space-time adaptive processing (STAP) for airborne radars allows the effect of clutter and jamming interference competing with targets to be reduced and higher signal to noise and interference ratios (SINR) to be obtained [1]-[2]. The efficacy of these adaptive filtering techniques is related to the goodness of estimation of the total interference covariance matrix (i.e. clutter plus noise and jamming), which in turn depends on the interference statistics and has to be estimated on the fly. In practice, the unknown interference covariance matrix is estimated in the ideal condition of statistically independent and identically distributed (IID) training data, which are assumed to be representative of the interference statistics in a cell under test. When working with real data, in presence of discrete clutter returns, targets, jamming and in nonstationary and/or heterogeneous clutter scenario, effectiveness of STAP can be highly reduced [3]. Therefore a number of algorithms, the socalled nonhomogeneity detectors (NHD), have been developed in recent years [4]. These detectors are employed to identify and excise outliers thereby improving the performance of covariance matrix estimation algorithms by using only homogeneous training data sets. However NHDs are only partially effective when the training data used to estimate the clutter covariance matrix contains two or more homogeneous areas. This contribution addresses this problem. Real data sets are often characterized by a collection of quasi-homogeneous adjacent subsets. For example, there can be an area covered by grass and then another with trees followed by a river or a sandy beach followed by the sea. The aim of this paper is the definition of an algorithm which discriminates between adjacent areas on the ground which generate clutter with different, but known, statistics. This approach is not proposed as a replacement for statistical nonhomogeneity detection, but as a preprocessing step to enhance the ability of these detectors to filter other types of nonhomogeneities such as targets or discrete clutter returns. The effects of clutter edges on covariance matrix estimates were first analyzed by Melvin using different clutter powers and mean-square velocity spreads of clutter 
Doppler spectra [3]. Melvin used the normalized signal-to-interference-plus-noise ratio, also called SINR loss, for evaluating STAP performance in heterogeneous environment. In this paper we characterize the normalized SINR as a function of the number of training samples. In addition we compare SINR losses for heterogeneous training data to those obtained when DHD is applied in conjunction with STAP. Due to the unpredictability of heterogeneity characteristics, this analysis extends the results in [3] for practical scenarios of operational interest.

\section{STAP and DHD in heterogeneous environment}

Consider a simulated clutter data set originated by an ideal terrain area including two different and adjacent regions, say "region A" and "region B". Consider an airborne radar flying above region $\mathrm{A}$ and moving towards region $\mathrm{B}$. In the Different Homogeneity Detector (DHD) algorithm, data from an array antenna operating over a narrow band of frequencies and a basic airborne radar scenario are considered: L-band, pulse Doppler, sidelooking radar with no platform crab and a velocity of $100 \mathrm{~m} / \mathrm{s}$; broadside transmit direction, uniform weighting on the transmit illumination function; ten half-wavelength spaced spatial channels $(\mathrm{N}=10$, $d=\lambda / 2$ ), each with identical cosine squared illumination functions and $180^{\circ}$ null beamwidths. Each channel measures fifteen pulses $(M=15)$, with NM samples collected over $L$ range cells. In addition, we compute all data for a single slant range of $50 \mathrm{~km}$, thereby avoiding the range-dependent influence of the two-way antenna pattern, and at a platform height of $9 \mathrm{~km}$. The number of clutter patches on a clutter ring is set to $N_{c}=180$, and $\beta=2 v_{a} T / d=1$. Suppose that, initially, there are no outliers in the simulated data set. It is known that received radar signals from target-free areas can be modelled as Gaussian processes. The in-phase and quadrature components are measured to fully represent the baseband complex envelope of the received signal. The I and Q components are stored as real and imaginary numbers in complex valued samples of the complex envelope. For clutter signals formed from the summation of many independent clutter patches, the central limit theorem can be invoked. The DHD problem can be formulated as follows. Consider data from the first pulse and the first spatial channel. These $L$ complex samples are assumed to be Independent circular complex Gaussian random variables with zero mean and variance $\sigma_{A}^{2}$ or $\sigma_{B}^{2}$. The square modulus of the complex samples, $\left|z_{k}\right|^{2}, k=1, \ldots, L$, is exponentially distributed with mean (and standard deviation) $\eta_{A}=2 \sigma_{A}^{2}$ or $\eta_{B}=2 \sigma_{B}^{2}$. Suppose that the parameters $\eta_{A}$ and $\eta_{B}$, corresponding to region A and B respectively, are known (they could be estimated from data) and that $\eta_{A}<\eta_{B}$. Consider a moving window which averages the square modulus of $P$ samples: $\bar{x}=\sum_{l=i}^{i+P-1}\left|z_{l}\right|^{2} / P ; i=1,2, \ldots, N-P+1$. Since $x_{l}=\left|z_{l}\right|^{2}$ are independent random variables, if $P$ is not too small, the sample mean is approximately Gaussian and its mean is $\eta_{A}$, when all $x_{l}$ comes from region $\mathrm{A}, \eta_{B}$ when all $x_{l}$ comes from region $\mathrm{B}$, and linearly increases from $\eta_{A}$ to $\eta_{B}$ when the moving window goes from region $\mathrm{A}$ to $\mathrm{B}$ (linearly decreases from $\eta_{B}$ to $\eta_{A}$ when the moving window goes from region B to A). It is known that the variance of the sample mean decreases with increasing sample size. Thus, to detect the border between regions $\mathrm{A}$ and $\mathrm{B}$, we calculate the sample mean and compare it with the threshold $\lambda=\left(\eta_{A}+\eta_{B}\right) / 2$. The performance of this methods depends on the window size $P$. It is observed that other independent samples could be obtained from the other channels and pulses. In any case DHD must be performed before STAP, and a window size of sixty 
pulses $(P=60)$ from the first pulse and the first channels allows the sample variance to be sufficiently reduced and the border detection to be performed before Space-time processing. With this result, if there is a target competing with the clutter in region $\mathrm{A}$, all the cells from region $\mathrm{B}$ can be excised. This permits STAP to be performed using only homogeneous training data. In presence of outliers, this permits NHD to be employed only for quasihomogeneous secondary data. Similar results can also be obtained using the Generalized Inner Product (GIP) or the test statistics characterizing the Modified Sample Matrix Inversion (MSMI) test or the Sample Matrix Inversion algorithm (SMI) [4]. This permits STAP to be performed simultaneously with NHD and also with DHD using efficient algorithms.

\section{Theoretical considerations and experimental results}

In this section, simulated airborne radar data are used to illustrate the performance improvements achieved when DHD is used in conjunction with STAP in heterogeneous environment. Detection of targets in clutter involves a decision between two hypotheses

$H_{0}: \mathbf{x}_{\mathbf{k} / \mathbf{H}_{0}}=\mathbf{x}_{\mathbf{c}, \mathbf{k}}+\mathbf{x}_{\mathbf{n}, \mathbf{k}}, H_{1}: \mathbf{x}_{\mathbf{k} / \mathbf{H}_{1}}=\alpha \mathbf{s}_{\mathbf{T}}+\mathbf{x}_{\mathbf{k} / \mathbf{H}_{0}}$,

for the k-th realization, where $\mathbf{x}_{\mathbf{n}, \mathbf{k}}$ is the receiver noise vector, $\alpha$ is a complex constant of unknown amplitude and uniformly distributed phase, and $\mathbf{x}_{\mathbf{c}, \mathbf{k}}$ and $\mathbf{s}_{\mathbf{T}}$ are the space-time snapshots of clutter and target, respectively. It is known that the linear transversal filter $y_{k}=\mathbf{w}_{\mathbf{k}}^{\mathbf{H}} \mathbf{x}_{\mathbf{k}}$, with $\mathbf{w}_{\mathbf{k}}=g \mathbf{R}_{\mathbf{k}}^{-1} \mathbf{s}_{\mathbf{T}} ; \quad \mathbf{R}_{\mathbf{k}}=E\left\{\mathbf{x}_{\mathbf{k} / \mathbf{H}_{0}} \mathbf{x}_{\mathbf{k} / \mathbf{H}_{0}}^{\mathbf{H}}\right\}, \quad\left(\mathbf{w}_{\mathbf{k}} \in C^{M N \times 1}\right.$ and $g$ is an arbitrary constant) maximizes the output SINR [1-2]. The covariance matrix $\mathbf{R}_{\mathbf{k}}$ is usually unknown and is commonly replaced with its maximum likelihood estimate (MLE) $\hat{\mathbf{R}}_{\mathbf{k}}=\frac{1}{L} \sum_{\substack{r=1 \\ r \neq k}}^{L} \mathbf{x}_{\mathbf{r}} \mathbf{x}_{\mathbf{r}}^{\mathbf{H}}=\frac{\mathbf{X X}}{L}$. The adaptive weight vector is then given by $\hat{\mathbf{w}}_{\mathbf{k}}=\hat{\mathbf{R}}_{\mathbf{k}}^{-1} \mathbf{S}_{\mathbf{T}}$. The performance of this approach, also called the Sample Matrix Inversion (SMI) algorithm, depends on how closely $\hat{\mathbf{R}}_{\mathbf{k}}$ approximates $\mathbf{R}_{\mathbf{k}}$. The performance as a function of the number of training samples has been theoretically characterized in a well-known paper by Reed, Mallet, and Brennan [5], using the normalized SINR. We indicate with $\mathbf{R}_{\mathbf{c}, \mathbf{n}}=\mathbf{R}_{\mathbf{c}}+\mathbf{R}_{\mathbf{n}}$ the clutter plus noise covariance matrix, $\mathbf{R}_{\mathbf{n}}=\sigma_{n}^{2} \mathbf{I}_{M \times N}, \mathbf{R}_{\mathbf{c}}=\mathbf{E}\left\{\mathbf{x}_{\mathbf{c}} \mathbf{x}_{\mathbf{c}}^{\mathbf{H}}\right\}=\sigma_{n}^{2} \sum_{k=1}^{N c} \xi_{\mathbf{k}} \mathbf{V}_{\mathbf{k}} \mathbf{V}_{\mathbf{k}}^{\mathbf{H}}=\mathbf{V} \boldsymbol{\Sigma}_{\mathbf{c}} \mathbf{V}^{\mathbf{H}}$, $\mathbf{V}_{\mathbf{k}}$ is the matrix of the clutter space-time steering vectors, $\boldsymbol{\Sigma}=\sigma_{n}^{2} \operatorname{diag}\left(\xi_{1}, \ldots, \xi_{N c}\right), \sigma_{n}^{2}=1$, and $\xi_{k}$ represents the clutter-to-noise ratio per element and per pulse, Data snapshots are generated as $\mathbf{x}_{\mathbf{c}, \mathbf{n}}=\mathbf{R}_{\mathbf{c}, \mathbf{n}}^{1 / 2} \mathbf{b}_{\mathbf{k}}$ [6], where $\mathbf{b}_{\mathbf{k}}=(1 / \sqrt{2})(\operatorname{randn}(N M, 1)+j \times \operatorname{randn}(N M, 1))$, and 'randn' indicates the Gaussian random number generator. Normalized SINR is a random variable with a beta distribution [5]. We use Monte Carlo simulation to calculate its sample mean, as a function of the number of data snapshots $(L)$, on $N_{m c}=400$ Monte Carlo trials. Fig. 1 displays results obtained for edge effects and the following scenario (see [3]): $\sigma_{v, A}=0 \mathrm{~m} / \mathrm{s}$ (primary data, region A) $\sigma_{v, B}=1.2 \mathrm{~m} / \mathrm{s}$ (secondary data, region B: sea region with a surface wind speed greater than $10 \mathrm{~m} / \mathrm{s}$ [7]), and $\mathbf{R}_{\mathbf{c}, \mathbf{A}}=100 \mathbf{R}_{\mathbf{c}, \mathbf{B}}$. The presence of Intrinsic Clutter motion (ICM) determines a temporal decorrelation [1] which can be taken into account through the Hadamard product of the covariance matrix with the matrix $\mathbf{A}=\operatorname{toeplitz}(\rho(0), \ldots, \rho(M-1)) \otimes \mathbf{1}_{\mathbf{N}}$, where $\mathbf{1}_{\mathbf{N}}$ is a $N \times N$ matrix of ones and 
$\rho(i-j)=\exp \left(-(i-j)^{2} T^{2} 8 \pi^{2} \sigma_{v}^{2} f_{0}^{2} / c^{2}\right)$. Fig1 shows the results when the beam is steered broadside, $L=N M, 1.5 N M, 2 N M, 3 N M, 4 N M, 5 N M$, the target normalized Doppler frequency is $\bar{f}_{d}=0.3$, with DHD (all training data from region A) and without DHD $(L / 2$ secondary data from region $\mathrm{A}$ and $L / 2$ from region $\mathrm{B}$ ). We observe that, with target free training data, DHD data elaboration allows the normalized SINR averaged on $N_{m c}$ Monte Carlo trials to be indistinguishable from the analytic solution (RMB rule): $E\{N \operatorname{SINR}\}=(L+2-N M) /(L+1)$. Fig. 2 shows the same results in the case of multiple borders (A, B, A, B, A) with DHD (all training data from region A) and without DHD ( $L / 10$ secondary data from region A and $9 L / 10$ secondary data from region $\mathrm{B})$. DHD may be easily extended to the case of multiple different homogeneous adjacent clutter regions as a multihypothesis decision problem.

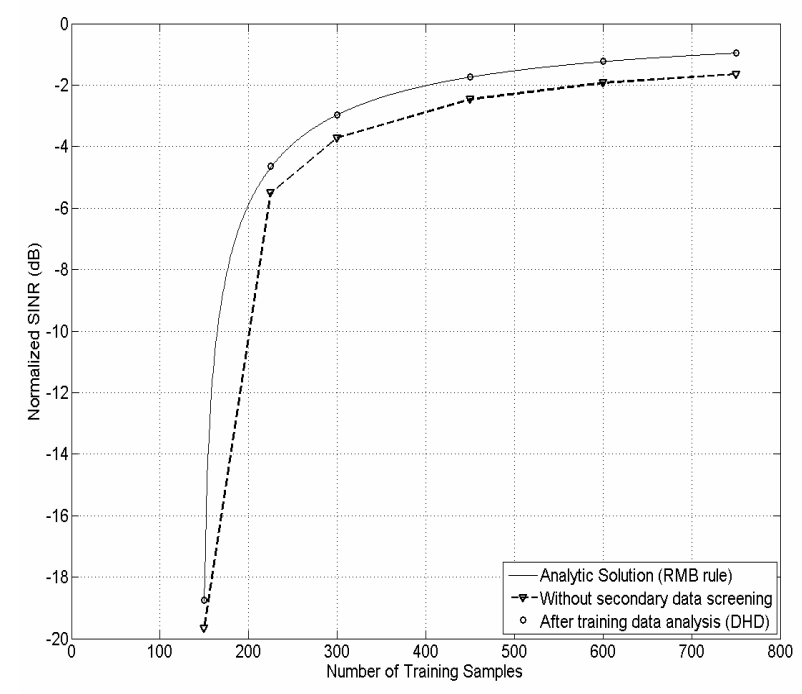

Fig. 1

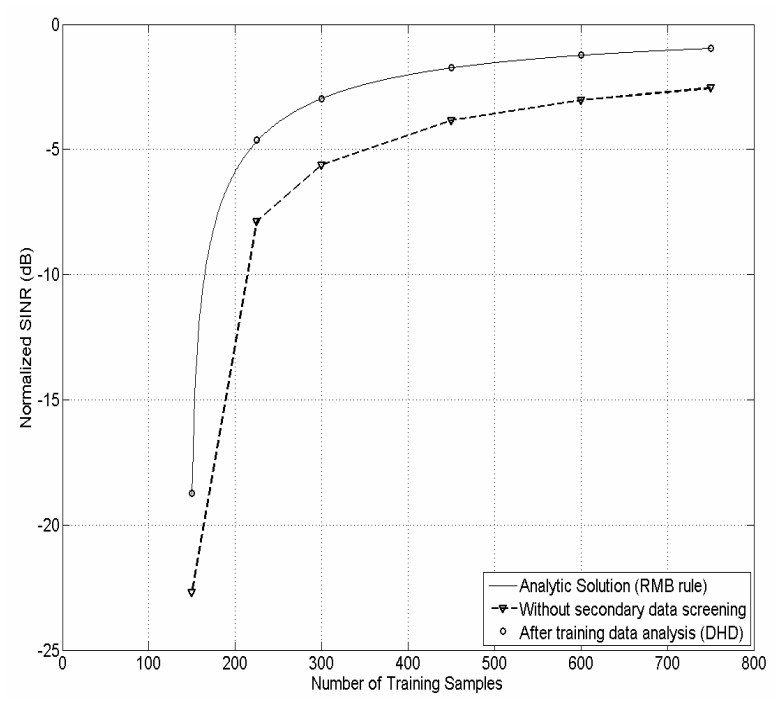

Fig. 2

\section{Reference:}

[1] Ward, J., "Space-time adaptive processing for airborne radar," Tech. Report 1015, Lincoln Laboratory, Dec. 1994.

[2] Klemm, R., Principles of Space-Time Adaptive Processing, IEE, UK, May 2002.

[3] Melvin, W. L., "Space-time adaptive radar performance in heterogeneous clutter," Aerospace and Electronic Systems, IEEE Transactions on, Volume 36, Issue 2, April 2000, 621 - 633.

[4] Wicks, M. C., Melvin, W. L., Chen, P., "An efficient architecture for nonhomogeneity detection in Space-Time Adaptive Processing airborne early warning radar," Radar 97 (Conf. Publ. No. 449), 14-16 Oct. 1997, Page(s):295 - 299.

[5] Reed, I. S., Mallett, J. D., and L. E. Brennan., "Rapid convergence rate in adaptive arrays," IEEE Trans. Aerosp. Electron. Syst., AES-10(6):853-863, November 1974.

[6] Hiemstra, J. D., "Robust Implementations of the Multistage Wiener Filter," Ph. D. thesis, Virginia Polytechnic Institute and State University, Apr. 2003.

[7] Fred E. Nathanson, Radar Design Principles, McGraw-Hill Book Co., New York, 1991. 